\title{
Le journal personnel comme pièce du dossier génétique
}

\section{Françoise Simonet-Tenant}

\section{(2) OpenEdition}

1 Journals

\section{Édition électronique}

URL : http://journals.openedition.org/genesis/425

DOI : $10.4000 /$ genesis. 425

ISSN : 2268-1590

Éditeur :

Presses universitaires de Paris Sorbonne (PUPS), Société internationale de génétique artistique littéraire et scientifique (SIGALES)

Édition imprimée

Date de publication : 1 janvier 2011

Pagination : 13-27

ISBN : 978-2-84050-749-9

ISSN : 1167-5101

\section{Référence électronique}

Françoise Simonet-Tenant, « Le journal personnel comme pièce du dossier génétique », Genesis [En ligne], 32 | 2011, mis en ligne le 01 juillet 2013, consulté le 19 avril 2019. URL : http:// journals.openedition.org/genesis/425; DOI : 10.4000/genesis.425 


\section{Le journal personnel comme pièce du dossier génétique}

Françoise Simonet-Tenant

$\mathrm{O}$

$\mathrm{n}$ a longtemps réduit le journal personnel à une simple source de renseignements historiques et biographiques. Peu à peu on en est cependant venu à le considérer comme un objet d'étude à part entière et à concevoir qu'il pouvait aller bien au-delà d'un geste d'improvisation aveugle et sans envergure. Si l'on considère le rapport du journal personnel à la genèse de l'écriture d'une œuvre autre que lui-même, le journal ne se limite pas nécessairement à un simple discours d'escorte. Il est cela certes, mais aussi beaucoup plus. Nous souhaiterions montrer qu'il n'assure pas seulement une fonction de commentaire à distance mais qu'il peut devenir le théâtre même de cette genèse, avec ses balbutiements, ses piétinements, ses errances et ses fulgurances. De l'œuvre poursuivie, il peut offrir la genèse en spectacle.

La nécessité de l'analyse nous conduira à distinguer la diversité de ses rôles, distinction didactique mais qui masque la réalité d'un objet polyvalent, riche de formes d'écritures diverses qui peuvent intervenir à des moments très différents du processus génétique de l'œuvre. Le journal personnel peut être un des supports et des lieux où s'effectue la genèse, de la phase prérédactionnelle à la phase prééditoriale. Champ de bataille des débuts d'œuvres, réceptacle de bribes encore informes, il est aussi, dans certains cas, un véritable atelier d'écritures. L'on peut même imaginer que le journal serait un lieu idéal de genèse... ou, tout du moins, un support commode d'étude génétique dans la mesure où il livre les pièces de la genèse datées, et donc aisées à classer. La réalité est néanmoins plus complexe, les journaux personnels abritant le plus souvent non pas une genèse d'œuvre dans son intégralité mais seulement certains de ses moments, variables selon les auteurs ; qui plus est, il n'est pas rare que certains diaristes tiennent leurs journaux sur des cahiers écrits en parallèle, et la genèse d'une même œuvre peut donc être dispersée aux mêmes dates sur des supports diaristiques différents.

\section{La fulgurance de l'idée}

Le journal personnel, cocon scriptural, est un espace graphique idéal pour abriter les idées fulgurantes et séduisantes qui n'ont pas encore trouvé le lieu et le temps de leur réalisation mais dont on veut garder la trace. L'idée fulgurante est labile, et il est difficile de garder la trace des incessants rebonds d'une pensée qui passe toujours à autre chose. Or l'écriture diaristique, initialement intransitive, n'est pas entravée par l'inhibition que peut susciter le projet d'une écriture lisible à communiquer. On peut, dans le journal, inscrire ses idées les plus chimériques, sans autocensure, dans l'attente d'une éventuelle mise en œuvre. Le journal, lieu d'écriture dynamisé par la perspective d'un perpétuel lendemain, se prête bien à la verbalisation d'une pensée en mouvement. Dans un article récent, Sophie Hébert oppose les genres du journal et du carnet, le journal étant réservé à l'événementiel et au dérisoire de

La phase

prérédactionnelle : le journal, espace d'écritures multiples 
la vie tandis que le carnet, plus noble, consignerait les idées et serait plus proche de l'essai que du journal. Qui plus est, le journal « restitue la pensée dans l'intégralité de sa progression et non dans ses moments forts », et il obéit à une « logique d'écoulement, de "bavardage" dans laquelle le carnétiste ne se reconnaît pas $^{1}{ }$. Cette distinction (et hiérarchisation) semble artificielle, et l'on rappellera que les diaristes sont (ou ont été) nombreux à tenir des journaux sur des carnets, support portatif commode... mais surtout que le journal personnel n'est pas uniquement voué au bavardage narcissique : peuvent aussi s'y inscrire des idées et même des événements de pensée ! Tenons-nous-en à la prudente observation de Louis Hay selon laquelle des « brèches » apparaissent dans la cloison qui sépare journaux personnels et carnets d'écrivains, «trop nombreuses et trop grandes pour ne pas provoquer quelque doute 2 » et n'oublions pas la sage et ouverte définition d'Amiel qui fait du journal le lieu des acta, sentita et cogitata.

Les journaux gardent la trace initiale d'idées qui font leur chemin - parfois semé d'obstacles -, même lorsque l'exécution du projet est bien éloignée de sa formulation initiale. Il en va ainsi du désir d'Henri-Pierre Roché de fixer verbalement sa passion pour Helen Hessel. Dans le roman Jules et Jim, publié en 1953, popularisé par le film de François Truffaut (1961), Henri-Pierre Roché a transposé l'histoire de son amitié pour l'écrivain allemand Franz Hessel et de sa passion pour Helen Hessel, amour tourmenté qui a duré treize ans et s'est terminé tragiquement en 1933. En 1920, Franz Hessel a invité Henri-Pierre Roché à lui rendre visite au domicile familial à Hohenschäftlarn. Très vite, une relation passionnelle se noue, sous le regard compréhensif de Franz, entre sa femme et son ami. Une première séparation des amants - Roché revient à Paris d'octobre 1920 à février 1921 - donne lieu à une correspondance active et à la réalisation d'un projet suscité par Roché, dont il a inscrit, sous forme télégraphique, les prémices dans son propre journal : «Idée d'écrire en roman notre histoire à nous quatre : Hélène, Franz et Bobann et moi en quadruple Tagebuch ${ }^{3}$. » Le 5 octobre, Roché transforme le projet. L'histoire à quatre points de vue devient une histoire à deux points de vue : « nous écrirons ensemble, parallèlement notre histoire à nous deux, sous forme de "Journal" simultané, chacun ignorant ce que l'autre écrit. - Cela ferait donc un amour vu à la fois des deux côtés, ce qui je crois n'a jamais été fait, avec une netteté et une franchise comme nous les aurons 4 ». Ce diariste acharné qui n'a cessé, de 1902 à avril 1959, de remplir de petits agendas de poche5, demande donc à Helen de rédiger un journal, ou plus exactement, un récit rétrospectif, daté et journalier de leur histoire d'amour. Helen s'exécute : elle rédige en trois langues (anglais, français, allemand), entre octobre 1920 et février 1921, un journal rétrospectif qui couvre la période de juillet à octobre 1920. Roché, initiateur du récit journalier d'Helen

1. Sophie Hébert, Recto/Verso, n 5, décembre 2009, p. 5 (article consultable en ligne : <www.revuerectoverso. com>).

2. Louis Hay, «L'amont de l'écriture », Carnets d'écrivains. Hugo, Flaubert, Proust, Valéry, Gide, du Bouchet, Perec, t. I, Paris, Éditions du CNRS, coll. «Textes et Manuscrits », 1990, p. 13.

3. Henri-Pierre Roché, Carnets, Marseille, Éditions André Dimanche, 1990, p. 66 (25 septembre 1920). Tagebuch: journal intime ; Bobann: Johanna Hessel, née en 1884, sœur d'Helen, avec laquelle Franz a une aventure fugace. 4. Ibid., p. 76 (5 octobre 1920).

5. Ibid., p. XXXI : «Henri-Pierre Roché n'a cessé, jour après jour, année après année, de noircir de sa petite écriture ses minuscules agendas de poche qu'il transcrit et développe ensuite, avec une écriture beaucoup plus aérée et formée, dans de grands Date Books anglais cartonnés. » 
Hessel, le lit avec admiration, mais ce texte qui a pris son autonomie ne correspond pas à ce qu'il attendait, et il ne parviendra pas à en faire, comme il l'avait prévu, le matériau d'une forme polyphonique. Néanmoins l'idée «d'écrire en roman » leur histoire sommeille sans disparaître. La nouvelle de la mort de Franz Hessel en 1941 la ranimera : Roché compose alors un roman hétérodiégétique, bien éloigné de la forme littéraire initialement prévue. L'on a pu avancer que le journal d'Helen avait fonctionné tel un lointain hypotexte qui aurait nourri le roman6, lequel se termine d'ailleurs sur une reconnaissance cryptée de l'indocile récit de l'indomptable Helen : «Le Journal intime de Kate a été retrouvé et paraîtra peut-être un jour? ${ }^{7}$. »

\section{Écritures obsessives}

Il est des idées et des images, des obsessions intellectuelles et poétiques, qui ne s'imposent pas sur le mode de la fulgurance mais qui s'inscrivent progressivement dans le journal jusqu'à dessiner les traits privilégiés d'un imaginaire. L'œuvre de Cocteau, diariste et poète, donne des exemples de ce travail souterrain. Il tient pendant le tournage de La Belle et la Bête un journal intéressant à plus d'un titre dont l'écriture commence le 26 août 1945, veille du premier jour de tournage, et s'achève le 1er juin 1946, lendemain de la première projection du film. Lorsque le tournage se termine, Cocteau dispose, en janvier 1946, d'une belle collection d'images fixées sur la pellicule, et le diariste note : «J'ai fini. C'est dire que je commence. J'ai récolté des images ${ }^{8}$. » L'on peut observer que Cocteau a fait non seulement provision d'images pour son film mais également pour son œuvre poétique. L'idée de sacrifice voire d'expiation court comme un fil rouge tout au long du journal. Le diariste consigne les différentes souffrances qui l'accablent et qui le mettent à la torture : impétigo, eczéma, furonculose, urticaire, anthrax... Les descriptions des différents maux ponctuent le Journal d'un film :

Mon visage est devenu comme une carapace de gerçures, de ravines, de démangeaisons. Il me faut oublier ce masque et vivre dessous de toutes mes forces.

Toute ma figure se prend. Couverte de boursouflures, de croûtes, de je ne sais quel sérum acide qui coule et qui me ravage les nerfs.

Ravagé d'urticaire, de gourme, de maux de toute sorte, je m'acharne, je continue. Et j'aime cet acharnement ${ }^{9}$.

Le dire de la maladie occupe une place essentielle, et l'image donnée par Cocteau dans son journal est celle du poète héroïque et martyr qui expie ce qu'il fait endurer aux acteurs, et en particulier à Jean Marais, qui doit subir cinq heures de maquillage et un masque plus qu'inconfortable pour se transformer en «Bête ». La conception de La Crucifixion (long poème composé de vingt-cinq strophes hétérométriques) est contemporaine du

6. Voir Xavier Rockenstrocly, Henri-Pierre Roché. Profession : écrivain, thèse sous la direction de Claude Martin, Université Lumière-Lyon II, 1996, deuxième partie, chap. II, p. 184-235, consultable sur <http://xavier.rockenstrocly.free.fr>.

7. Henri-Pierre Roché, Jules et Jim, Paris, Gallimard, coll. « Folio », 1980, [1953], p. 243.

8. Jean Cocteau, La Belle et la Bête. Journal d' un film, Paris, J.-B. Janin, 1946, p. 217 (11 janvier 1946).

9. Ibid., p. 86 (26 septembre 1945), p. 90-91 (28 septembre 1945), p. 105 (7 octobre 1945). Voir aussi p. 118, p. 121, p. 123 , p. 130 , p. 131 , p. $209 \ldots$ 
tournage du film, et l'on ne peut manquer de noter, dans le Journal d'un film, certaines observations :

Dimanche

Lorsque les fausses teintes commencent et que les nuages bougent mystérieusement de telle sorte que l'aide-opérateur, qui les observe avec son verre jaune, ne peut jamais savoir si ses pronostics sont justes, je me couche sur l'herbe, je ferme les yeux et mon poème (La Crucifixion) me travaille. Il m'emporte si loin, n'ayant aucun contact avec ce qui m'entoure, que j'ai l'air, si le soleil approche et que le guetteur l'annonce, de me réveiller en sursaut ${ }^{10}$.

Les affres de la création vécues sur un mode agonique par le moi-peau trouvent dans le journal un premier lieu d'expression, mais s'esquisse par là même l'image d'une figure christique qui va inventer sa voix lyrique dans le poème en devenir. Les notes diaristiques peuvent sans doute être lues pour une part comme une forme préparatoire et peut-être inconsciente - du chant de douleur qui sera celui du poème. Sous la plume du diariste s'inscrit ainsi la déploration suscitée par « les clous », expression familière pour désigner les furoncles qui le tourmentent, mais le substantif polysémique appelle l'image des clous aux connotations christiques qui s'inscrira au cœur de la vingtième strophe du poème :

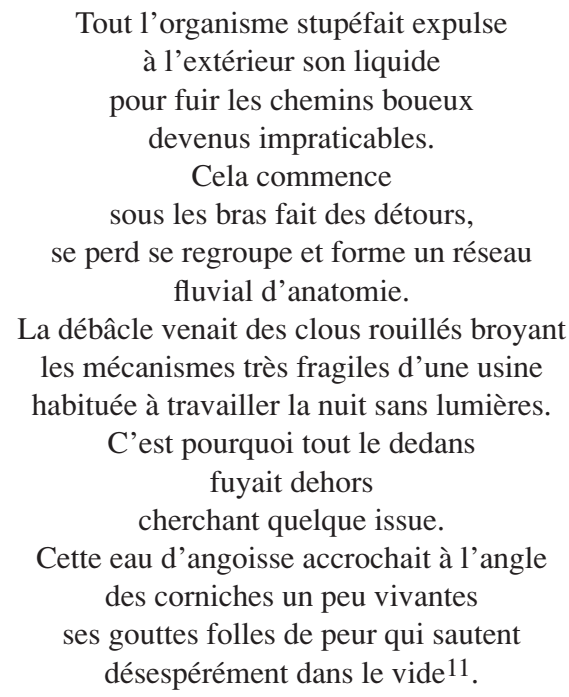

Avec Cocteau, le journal apparaît à la charnière d'une double genèse : à la fois livre de bord d'une genèse cinématographique et écriture en amont d'une genèse poétique, il permet la conversion d'images visuelles spectaculaires en images rhétoriques qui disent les tourments du corps et de l'âme.

10. Ibid., p. 40.

11. Jean Cocteau, Euvres poétiques complètes, Paris, Gallimard, coll. « Bibliothèque de la Pléiade », 1999, p. 699. 


\section{Écritures provisionnelles}

Le journal peut être utilisé également de manière très consciente et joue, dans certains cas, le rôle d'un précieux réservoir de l'œuvre. Chez Leiris, il est un élément fondamental de la genèse du texte autobiographique. Les cahiers sont en effet utilisés pour alimenter le fichier qui se trouve lui-même à la base du travail de La Règle du jeu, fichier qui porte l'empreinte de la formation ethnographique de Leiris : la tétralogie autobiographique, qui subvertit tout déroulement chronologique, est le fruit d'un bricolage poétique recourant au jeu des affinités souterraines entre les mots, à des techniques matérielles de découpages et de collages de textes, à l'utilisation de fiches où ont été notés rêves, réflexions, souvenirs... Le Journal, point d'articulation entre la vie et l'écriture, appartient donc aux fondations de l'édifice de La Règle du jeu. Tandis que le journal séquentiel collige les entrées sans tri, le fichier fonctionne tel un jeu de cartes - dont certaines sont constituées d'extraits d'entrées du journal - qui permet de multiples combinaisons.

Il est intéressant de considérer le cheminement d'une seule phrase du journal à Fourbis en passant par le fichier et d'en faire une microlecture rapide :

Avant de me coucher, je vais dans la cuisine pour manger et boire un peu. Adossé à un mur, je fume une cigarette et je pense à la mort ; mon regard tombe sur le compteur à gaz et sur la balance romaine 12 .

[...] et c'est un rien éclaircir non plus que raconter comment, étant légèrement ivre, j'allai un soir dans ma cuisine pour manger un quelconque bout de nourriture et ingurgiter un peu d'eau avant de me coucher, puis restai planté là, fumant une cigarette, le dos contre le mur, et pensai qu'il me faudrait fatalement mourir tandis que mon regard se posait tantôt sur le compteur à gaz, tantôt sur la balance Roberval à double plateau de cuivre13.

Du journal à l'autobiographie, la teneur du propos reste globalement la même à l'exception d'une circonstance - l'ivresse -, omise dans le journal et qui apparaît déjà dans le fichier, mais la mise en forme des faits présentés est modifiée : la parataxe, le style coupé diaristique et l'usage du présent laissent la place à une phase complexe dans l'autobiographie au passé simple. Cette dernière phrase, longue, s'est gonflée de plusieurs additions ( «étant légèrement ivre », « avant de me coucher », « et pensai qu'il me faudrait fatalement mourir », «à double plateau de cuivre »). La notation économique et prosaïque du journal donne lieu à une expansion que l'on ressent presque comme une redondance : « pour manger un quelconque bout de nourriture et ingurgiter un peu d'eau ». L'on peut imaginer que Leiris reprenant dans le fichier une phrase déjà copiée du journal la révise au moment de la faire passer dans le texte autobiographique, et en change radicalement le rythme et la tonalité. On passe de la brièveté sobre du constat à l'analyse distanciée (« et c'est un rien éclaircir non plus que raconter comment [...] »). L'importance accordée à des faits communs et menus - démarche qui intéressait Leiris chez Freud - est inhérente à la pratique diaristique. Néanmoins ces mêmes faits ressaisis par l'écriture autobiographique

12. Michel Leiris, Journal 1922-1989, Paris, Gallimard, 1992, p. 377 (7 février 1943). La version consignée par Michel Leiris dans le fichier est identique au texte du journal, à une seule exception : ajout entre crochets d'une expression entre «Avant de me coucher » et « je vais... » : <étant légèrement ivre>.

13. Michel Leiris, La Règle du jeu, Paris, Gallimard, coll. «Bibliothèque de la Pléiade », 2003, p. 354. 
semblent se lester d'un nouveau poids - effet suscité par l'hypertrophie syntaxique - et d'une valeur dramatique avec le spectre du décompte du temps qui reste et de l'image du jugement dernier.

\section{Écritures d'initialisation}

Le journal est délectable pour son auteur comme pour son lecteur quand il se fait rêverie, tout à la fois promesse et projet d'œuvre. Quelle plus belle promesse qu'un titre ? L'œuvre peut servir à recueillir des projets littéraires qui se réduisent parfois aux titres. Le Journal de Leiris pourrait s'appeler « rêverie sur les titres » tant ils sont nombreux à s'égrener au fil des pages : Les Fossiles de la mer, Les Carcasses de la faim, L'Homme sans honneur, Presque, Le vain recours, Le moins qu'on puisse dire, L'ultime prothèse, Charme rompu, Pourtant..., La triste entourloupette, Parce que, C'est-à-dire, Pied de la lettre... Nous n'en citons qu'un petit nombre ${ }^{14}$ : ces baptêmes poétiques semblent receler le plaisir de la liste inachevable, la jubilation née du rapt et du détournement des lieux communs, le jeu poétique de la trouvaille verbale, l'excitation de l'imagination aiguisée par des titres qui sont autant d'amorces d'œuvres... Comme le note Philippe Lejeune, «c'est dans les années quatre-vingt que la pratique va devenir plus fréquente, au point qu'on peut alors se demander [...] si ce n'est pas l'amorce d'un recueil : plus se rétrécit le temps qui reste pour écrire des œuvres, plus les titres fusent en gerbes $15 \ldots$ ». Parce que l'écriture diaristique, constitutivement, est toujours ouverte sur un lendemain, le journal personnel offre à son scripteur une garantie fantasmatique contre la mort. Un autre jour, une autre entrée : c'est l'inscription d'un nouveau titre, la promesse d'une autre œuvre et, peut-être plus encore, la quête d'une quintessence de l'œuvre car «Leiris avoue souvent rêver d'un livre où se résume le monde, dont le titre concentrerait en un mot toute la substance et tous les mots, rendant superflu tout développement, un mot-somme en quelque sorte qui congédierait la langue, un titre magique qui contiendrait l'Euvre16 ».

Le diariste peut aller au-delà du titre, et de promesse passer au projet quand il confie au journal l'argument (et parfois le plan) de l'œuvre à venir : « Dans GDP II, il sera question de la masturbation (de la femme comme abstraction, etc.) et du fétichisme (du bas, de la jarretelle, de la gaine etc. considérés abstraitement, indépendamment de la femme). Ceci est d'ailleurs à approfondir, à laisser croître au milieu d'un thème romanesque ${ }^{17}$. » Le diariste paraît ainsi consigner à destination du romancier une note de régie.

D'inscription de la trace de l'idée-événement en écritures obsessives, provisionnelles et initialisatrices, le journal, sans aucun doute, est un lieu propice à l'effervescence inventive. Parce que le journal est un lieu d'écriture où tout peut s'inscrire mais où rien n'est irrévocable, il est un espace libérateur où l'on peut se laisser aller, sans frein, à l'infini des possibles.

14. Pour une liste exhaustive, voir Philippe Lejeune, «Pistes, listes », Michel Leiris ou De l'autobiographie considérée comme un art, dir. Philippe Lejeune, Claude Leroy et Catherine Maubon, RITM, ${ }^{\circ} 31$, Université Paris X-Nanterre, 2004, p. 15-17.

15. Ibid., p. 13.

16. Marie-Paule Berranger, « Le Petit Leiris illustré », dans Michel Leiris ou De l'autobiographie considérée comme un art, op. cit., p. 32.

17. Raymond Queneau, Journal 1939-1940, Paris, Gallimard, 1986, p. 100 (10 décembre 1939). GDP II : Gueule de Pierre II, qui deviendra Les Temps mêlés. 


\section{Le journal, espace des brouillons romanesques}

Certains journaux, livrant la genèse d'œuvres en acte, sont de véritables ateliers d'écriture. C'est le cas du Journal de Kafka où le diariste s'essaie à maintes formes littéraires (formes brèves tel l'aphorisme, récits ou nouvelles, romans). Le Journal contient à la fois des ébauches de nouvelles (qui seront plus amplement développées dans la suite de l'œuvre), des nouvelles abandonnées, des récits achevés. Les romans ont leur place dans les cahiers mais sous des formes différentes : pour L'Amérique, il s'agit de parties du texte définitif du roman, consignées d'abord dans le Journal avant d'être reproduites telles quelles dans le roman définitif ; pour Le Procès, il ne s'agit que d'esquisses préparatoires 18 . Le journal est alors devenu un mixte inextricable de réalité et de fiction. Le journal joue-t-il un rôle différent selon la catégorie d'écrivains à laquelle on a affaire ? On sait que l'on distingue traditionnellement les écrivains à « programmation scénarique » et ceux à « structuration rédactionnelle », sans méconnaître que nombre d'écrivains combinent les deux démarches. Kafka, dont la démarche est essentiellement à structuration rédactionnelle, transforme son journal en brouillon. Si l'on prend le cas d'un romancier tel Roger Martin du Gard dont la démarche est largement programmative, le journal rythme et accompagne la genèse plus qu'il ne l'abrite. Élève naturellement nonchalant, éduqué à la rigueur de la construction par Louis Mellerio, professeur à Janson-de-Sailly chez lequel son père l'avait mis en pension, puis par sa formation de chartiste, Martin du Gard adapte à son métier de romancier les méthodes inculquées et se plie à la discipline du plan. En 1920, le romancier conçoit ce qui va devenir l'œuvre de sa vie, les Thibault, et le journal, qu'il tient depuis 1919, porte les traces de la maturation mentale du projet.

Depuis une huitaine de jours, il m'est arrivé cet événement considérable d'avoir une œuvre en train. Comme cela, sans raison; et même : en dépit de toutes les raisons que j'avais de ne pas concevoir une œuvre de cette sorte. Je suis tout étonné et comme charmé de l'éclosion mystérieuse. [...] C'est dimanche dernier, le 11 janvier, que je me suis aperçu que j'avais un sujet en tête : la vie de deux frères, bien distincts, l'un comme ceci ; l'autre comme cela... Je n'y ai presque pas fait attention. Deux jours après, tout s'était amplifié, dessiné, éclairci. Ce projet en l'air, comme bien d'autres, avait pris soudain un caractère inéluctable, sacré, impérieux ; il avait pris lentement et régulièrement possession de moi ; et je lui appartenais. [...] Chaque jour, et plusieurs fois par jour, comme pointent de petits bourgeons sur une branche, une idée se formule, sans effort, bien nette, et va d'elle-même prendre sa place autour du noyau de l'œuvre en gestation. Je la note, et n'y pense plus. Je ne cherche pas à travailler ${ }^{19}$.

Les notes du journal (ainsi que certaines lettres) jalonnent les étapes de ce «phénomène de gestation involontaire $20 »$, travail intime dont le journal balise la progression. En avril, il séjourne à Barbizon où il a « apporté des kilos de fiches à relire pour [son] livre21 ». En mai, il a rejoint son antre, la maison du Verger d'Augy, dans le Cher, pour bâtir au prix d'un « travail solitaire et forcené » son plan : «Pas une ligne n'est encore écrite. Mais je puis

18. Voir Florence Bancaud, Le Journal de Franz Kafka ou l'écriture en procès, Paris, CNRS Éditions, 2001, p. $225-252$.

19. Roger Martin du Gard, Journal II 1919-1936, Paris, Gallimard, 1993, p. 88-89 (18 janvier 1920).

20. Ibid., p. 89 (19 janvier 1920).

21. Ibid., p. 119 (14 avril 1920).

\section{La place du journal dans la phase rédactionnelle}


maintenant, avec une impression de sécurité totale, me donner successivement de toutes mes forces libres, à chaque période séparée. Je n'ai plus qu'à marcher, en suivant mon plan, sûr d'arriver au bout 22 . » Le 18 juillet 1920, d'une phrase qui a valeur d'un sceau, il note : «C'est aujourd'hui que j'ai commencé effectivement à écrire mon livre les Thibault23. »

À la lumière des deux exemples de Kafka et de Roger Martin du Gard, on peut élaborer une première hypothèse : pour l'écrivain à programmation scénarique, l'écriture diaristique serait avant tout un exercice de maîtrise qui enregistre les étapes, les événements et les progrès de la genèse, mais la genèse effective prend place sur un support disjoint du journal ; pour l'écrivain à structuration rédactionnelle, les supports d'écriture ne se voient pas attribuer de fonction spécifique et compartimentée, le flux créatif s'impose, indifférent au support.

L'hypothèse est sommaire, simplifiant une réalité complexe qu'un romancier aussi difficile à catégoriser qu'André Gide nous aide à nuancer. Pour Gide, l'écriture diaristique va être autant une écriture d'accompagnement qu'un soubassement de la fiction.

Premier roman gidien : Les Cahiers d'André Walter, publié en décembre 1890. Le récit se présente sous la forme d'un journal tenu en 1889 par un jeune homme, André Walter, qui s'est retiré en Bretagne après la mort de sa mère, laquelle lui a fait promettre de renoncer à épouser sa cousine Emmanuèle. Celle-ci a consenti à en épouser un autre. Le journal comprend deux cahiers : d'abord «Le Cahier blanc » où il couche des souvenirs et insère des fragments antérieurs de son journal (1886-1888) ; puis « Le Cahier noir », laboratoire du roman Allain qu'il est en train d'écrire. D'emblée se dégagent de l'univers romanesque du jeune Gide des traits promis à un bel avenir : donner l'impression au lecteur qu'il assiste au chantier d'un livre en cours (un trompe-l'œil génétique) et céder à la tentation paradoxale du roman-journal, le mariage de la carpe et du lapin autrement dit, celui d'un récit qui sait où il va et d'une écriture à l'aveuglette, ignorante de ses lendemains. De ce mariage improbable d'un récit tendu vers sa fin et d'une écriture par essence inachevable, Les Cahiers d'André Walter gardent l'empreinte. Gide formule explicitement, peu avant de s'atteler à l'écriture du récit, ce qui en fera la spécificité : «Dire, pour André Walter, l'absence de conclusion qui déroute24. »

Gide prête à son double la «manie écrivante 25 » qui est la sienne. C'est ainsi que se met en place un dispositif spéculaire vertigineux : le jeune Gide fait de son personnage un diariste, tient lui-même son journal au moment de la genèse des Cahiers d'André Walter et insère dans le journal fictif des fragments de son propre journal tenu entre août 1888 et juillet 1890 (plus d'une vingtaine de fragments parfois relativement amples). Quand Gide s'apprête à se mettre au travail, la résolution s'inscrit dans le journal, plus ferme d'avoir été écrite : « Il faut faire Allain. Examen d'André Walter. (Commencer dès à présent à rassembler des notes.) [...] Il faut travailler avec acharnement, d'un coup, et sans que rien vous distraie ${ }^{26}$. » Seules quelques entrées dispersées et brèves s'inscriront les semaines suivantes dans le journal jusqu'à la publication du roman en décembre. À compter de mai 1890, Gide tient un cahier préparatoire aux Cahiers d'André Walter où il couche à la fois des réflexions sur son

22. Ibid., p. 134 (26 mai 1920).

23. Ibid., p. 147 (18 juillet 1920).

24. André Gide, Journal 1887-1925, Paris, Gallimard, coll. « Bibliothèque de la Pléiade », 1996, p. 119 (8 mai 1890).

25. Expression chère aux deux diaristes Valery Larbaud et Paul Léautaud.

26. André Gide, Journal 1887-1925, op. cit., p. 119 (8 mai 1890). 
livre à venir et des réflexions prêtées à son personnage, Allain. Dans une intéressante note de régie, il observe : «Il faut reproduire mon premier morceau, le tout premier du Cahier gris, mais sans les longueurs, il faut peiner le style, châtier indéfiniment la forme 27. » L'on remarque le curieux usage en ce sens de la construction transitive du verbe «peiner », et l'on ne peut s'empêcher de rapprocher cette note de l'analyse postérieure faite par Roland Barthes dans « Délibération 28 », interrogation sceptique sur le journal personnel, partagée entre fascination et dévaluation d'une écriture jugée « inessentielle », « non nécessaire » et « inauthentique ». Néanmoins Barthes s'arrange pour proposer, in extremis, une rédemption paradoxale au journal : « il faudrait sans doute conclure que je puis sauver le Journal à la seule condition de le travailler à mort, jusqu'au bout de l'extrême fatigue, comme un Texte à peu près impossible. » «Peiner le style, châtier indéfiniment la forme » et « travailler [le journal] à mort » se font écho comme si le diariste portait la culpabilité d'une écriture qui puiserait à la vie et qui ferait l'impasse de la forme enfantée dans la peine... Gide saura néanmoins faire taire ce scrupule - aux résonances judéo-chrétiennes - et l'on observera que la plupart des fragments de son propre journal insérés dans Les Cahiers d'André Walter le sont presque sans modification. Pièce génétique essentielle du laboratoire de la fiction, le journal authentique ne saurait néanmoins être considéré comme un brouillon si l'on suit la démonstration d'Éric Marty et son analyse quasiment phénoménologique de la genèse des Cahiers d'André Walter :

Que signifient, en effet, ces insertions extrêmement nombreuses, ces recopiages littéraux du Journal « réel » dans le journal fictif ? Ils correspondent, en accord avec le désir de produire une autobiographie de fiction, au vœu de répéter l'expérience antérieure sous une forme romanesque. Il y a échec, car l'expérience du possible au sein du monde de la fiction ne peut être une expérience vive et immédiate, comme l'est celle du présent dans le Journal. Au travers du recopiage, l'expérience du Journal perd son authenticité - la présence toujours vive de son geste d'origine -, sans pour autant acquérir une valeur de fiction : le texte devient alors obsolète à lui-même, d'autant plus mal inséré qu'il est recopié. Il y a une articulation fondamentale entre les deux projets qui induisent deux positions de conscience saisies au sein de pratiques d'écritures antagonistes : l'une ne peut être le brouillon de l'autre, et la seconde une variation subjective de la première. [...] De sorte qu'entre le Journal tenu par Gide entre 1888 et 1890 et Les Cahiers d'André Walter, nous avons affaire à une genèse inachevée, et inaccomplie dans son intégralité : il y a plutôt ce que l'on appellerait une hybridation monstrueuse, fixation d'une chimère dont les morceaux ne peuvent se convenir 29 .

On peut ne pas partager l'avis d'Éric Marty sur l' « échec » de cette expérience scripturaire, mais l'on s'accordera avec lui pour donner au journal un statut de greffon dans Les Cahiers d'André Walter : l'hybridation génétique est-elle pour autant responsable du texte effectivement étrange des Cahiers d'André Walter, composite, où le lecteur a du mal à trouver sa place et sa position?

27. André Gide, Romans et récits. Euvres lyriques et dramatiques, t. I, Paris, Gallimard, coll. « Bibliothèque de la Pléiade », 2009, p. 140. Le « Cahier gris » désigne le cahier où Gide a tenu son journal d'octobre 1887 à octobre 1889.

28. Roland Barthes, « Délibération », Le Bruissement de la langue, Paris, Éditions du Seuil, 1984, p. $399-413$.

29. Éric Marty, « Gide et sa première fiction », dans L'Auteur et le manuscrit, dir. Michel Contat, Paris, Presses universitaires de France, 1991, p. 186. 
Le romancier renouvellera dans La Porte étroite un dispositif spéculaire analogue, de façon plus complexe encore et sans doute plus aboutie. L'inspiration des deux récits puise dans la même source : le dilemme qui tourmente Gide, déchiré entre amour sublimé et possession charnelle 30 . André Gide transpose dans La Porte étroite les années de crise morale et spirituelle qui ont précédé son mariage avec Madeleine. Ce récit sobre de facture classique, publié en 1909, résulte d'une genèse mentale et scripturaire, longue et complexe. La première mention du récit figure dans le journal du 13 octobre 1894: "Possibilité de détresse : l'âme qui croit avoir mal adoré. (Mort de Mlle Claire) 31 ». Gide commente luimême postérieurement dans son journal : «Devenu plus tard la Porte étroite. » Ce titre était lié à la reprise d'un projet où André Gide comptait retracer la mort d'Anna Shackleton, gouvernante puis amie de sa mère. Dès 1890, Gide avait confié à son journal : « Puis aussi l'étrange sentiment que l'âme d'Anna revive en Elle [Madeleine] $32 . . . »$ On comprend mieux alors que la figure d'Alissa dans La Porte étroite est une création fusionnelle derrière laquelle se profilent les ombres de Madeleine et d'Anna.

Créer, c'est «naviguer durant des jours et des jours sans aucune terre en vue 33 », dira Gide. La genèse est en effet une aventure de haute mer, on quitte un rivage connu sans savoir ce que le voyage réserve. C'est en ce sens que le journal peut être le livre de bord indispensable qui permet d'accompagner la traversée de l'inconnu, de consigner les changements de cap et de soutenir les efforts d'une aventure incertaine. Dans ce cheminement, non exempt d'impasses et de procédures dilatoires, le diariste soutient le romancier en recherche, et parfois en déroute. En juin 1905, André Gide entreprend la composition du récit dont le titre oscille entre La Porte étroite et La Route étroite et dont l'héroïne s'appelle Geneviève ; dans le même temps, il lit Goethe (Werther) et Dante (Vita Nuova); il reprendra à la fin de l'été la lecture des Pensées de Pascal. En juillet, il a lu son premier chapitre à Copeau. La poursuite du travail se révèle difficile : « Je passe des heures sur un groupe de phrases que je rebouleverserai le lendemain ${ }^{34}$. » À l'automne, nouvelle panne d'écriture. Deuxième départ en 1906 : pour se sortir de l'ornière, le romancier puise dans le terreau autobiographique. Le 29 mars 1906, il note dans son journal : « Je relis mes anciennes lettres à Em. que j' ai rapportées de Cuverville. En vain j'y cherche quelque aliment pour mon roman. Mais j'y contemple à nu tous les défauts de mon esprit. Il n'en est pas un seul contre lequel je ne m'irrite ${ }^{35}$. » $\mathrm{Ce}$ ne sont pas tant dans ses propres lettres que dans les lettres et le journal de Madeleine qu'il trouvera des aliments. En juin 1907, il reprend le récit, il note dans son journal : « Pour la quatrième fois complètement à neuf je reprends ce misérable livre, sur lequel j'ai déjà tant peiné. Les grands improvisateurs d'aujourd'hui crieraient à l'impuissance ou à la manie36. »

30. L'on ne manquera pas de noter ce court passage emprunté au début des Cahiers d'André Walter : «Après les ennuis cérémonieux qui distraient, nous avons communié ensemble. Emmanuèle était devant moi ; je ne 1'ai pas regardée, et, pour ne pas penser à elle et m'empêcher de rêver, je répétais : "Puisqu'il faut que je la perde, que je te retrouve au moins, mon Dieu, - et que tu me bénisses d'avoir suivi la route étroite" " (Romans et récits. Euvres lyriques et dramatiques, op. cit., p. 7).

31. André Gide, Journal 1887-1925, op. cit., p. 184.

32. Ibid., p. 116.

33. André Gide, Journal des faux-monnayeurs, Paris, Gallimard, coll. « L'imaginaire », 1995 [1927], p. 29.

34. André Gide, Journal 1887-1925, op. cit., p. 471 (31 juillet 1905).

35. Ibid., p. 515.

36. Ibid., p. 574 (22 juin 1907). 
Ce nouveau démarrage sera le bon ; les difficultés seront encore nombreuses mais après s'être arrêté sur le choix d'un récit autodiégétique, Gide maintiendra le cap. L'année suivante, Gide parvient en octobre au terme de son récit : «Atroce fatigue, qui dure même après le départ des Laurens. Pourtant j'achève La Porte étroite le 15 - et le 16 rase mes moustaches ${ }^{37}$. » Ce geste symbolique signe la victoire du romancier et la libération de l'homme qui, avec l'achèvement de son récit, est également parvenu (douloureusement) à liquider son passé et à s'émanciper de la loi maternelle et de l'héritage religieux oppressif 38 .

Pour se sortir de l'ornière où s'enlisait son récit en genèse, Gide a eu recours en 1906 au matériau autobiographique. Son propre journal mais aussi celui de Madeleine Rondeaux, sa cousine, ainsi que leurs lettres respectives constituent la mémoire écrite de la crise qui a précédé leur mariage. Néanmoins, André Gide ne puise pas dans cette mémoire écrite le seul argument du récit : il conduit le personnage d'Alissa à écrire lettres et journal, qu'il intègre dans le récit et où il insère des fragments de textes factuels authentiques. La vie des personnages eux-mêmes se réfléchit ainsi dans l'écriture, vouée à tout absorber dans l'univers gidien et appelée dans sa capacité de reflet et d'anamorphose à être le centre de l'intérêt romanesque. Si le diariste a eu recours à son propre journal pour procéder à une fictionnalisation de soi dans Les Cahiers d'André Walter, le romancier-écornifleur puise son matériau dans le journal d'autrui pour dynamiser une genèse romanesque difficile dans $L a$ Porte étroite 39 . Dans ces deux cas et à des titres différents, le journal constitue une pièce fondamentale du dossier génétique.

\section{Le journal comme atelier poétique}

Les exemples précédents de Gide, Kafka et de Roger Martin du Gard ne devraient pas nous laisser croire que le journal n'a partie liée qu'avec les genèses de romans. Durant les dernières années de son existence, le Journal de Catherine Pozzi abrite la gestation des rares poèmes dont elle a attendu qu'ils lui permettent de passer à la postérité. Nous prendrons pour seul exemple la genèse du poème «Ave » qui s'est étendue sur plusieurs mois.

Le premier état d'«Ave » ne comporte que deux vers où est déjà présente l'apostrophe à l'amour qui déterminera le mouvement du poème :

Amour qui m'as perdu, s'il se fait que je meure

D'avoir vu mes pays exister sous ton $\operatorname{ciel}^{40}$

37. Ibid., p. 602-603.

38. Voir André Gide, Romans et récits, Euvres lyriques et dramatiques, op. cit., p. 1433-1435 (notice de Pierre Masson).

39. Selon Pierre Masson, dans la dernière et belle édition qu'il a donnée de La Porte étroite dans la Pléiade, on voit Gide « piquer ici et là quelques phrases dans le journal ou les lettres de Madeleine, utilisant les réticences que celle-ci manifesta envers le mariage durant quatre ans, de 1891 à 1895 » (op. cit., p. 1433). L'expression «piquer ici et là » minimise des emprunts qui ne sont pas négligeables : six emprunts au journal de Madeleine et quatre à ses lettres dont la fameuse lettre sur le « triste revoir » de Jérôme et Alissa qui est intégralement calquée sur la lettre du 29 décembre 1894 envoyée par Madeleine à son cousin. Voir Françoise Simonet-Tenant, « André Gide et Madeleine Rondeaux : une mosaïque textuelle », Le Moi et ses modèles. Genèse et transtextualités, dir. Véronique Montémont et Catherine Viollet, Louvain-la-Neuve, Academia-Bruylant, 2009, p. 67-83.

40. C. Pozzi, Journal inédit, 1er juillet 1928, BnF. 
Catherine Pozzi travaille longuement son poème en janvier 1929, comme en témoignent les différentes versions d' «Ave » présentes dans les cahiers. Le poème donne lieu à un intense travail de réécriture qui se déroule simultanément en janvier 1929 sur trois supports : le cahier numéroté XXIV du journal qui va du 14 novembre 1928 au 9 février 1929 ; un cahier commencé en 1911, vite interrompu qui, selon une note de la main même de Catherine Pozzi, « lui servira de registre d'exercices 17 ans après 41 »; un troisième cahier qui va de décembre 1927 à mars 1929, qui constitue essentiellement un cahier de travail. À la date du 31 décembre 1928-1er janvier 192942 apparaît le moule strophique, présent dans la version définitive du poème, selon lequel chaque strophe est constituée de quatre décasyllabes et d'un tétrasyllabe. Dès les 9-10 janvier 1929, les trois premières strophes ont acquis leur forme quasiment définitive et la structure circulaire d' « Ave » est fixée - la même expression « Très haut amour » ouvre et clôt le texte. En revanche, ce qui deviendra les quatrième et cinquième strophes subit de nombreuses réécritures. Le 4 février 1929, Catherine Pozzi note dans son journal : «La nuit, j'ai repris le poème et repris encore le lendemain et enfin à 7 heures, il a été fini. C'était hier. Je l'ai envoyé à Paulhan ce matin ${ }^{43}$. » Dans cette version envoyée à Jean Paulhan, la dernière strophe est la suivante :

En quel futur, plus sûr que ce mirage,

De mille instants déliés sans retour

Referez-vous mon nom et mon image,

Cour de l'esprit, pour le nouveau voyage,

Très haut amour 44 .

Le circonstanciel y affleure. En témoigne une observation explicite, consignée dans le Journal, où la diariste se plaît à imaginer les réactions de Paul Valéry, surnommé « Hell », à la lecture d' "Ave », et elle interprète certains des vers écrits à la lumière de la relation passionnelle qui a été la leur : «Quelqu'un, Hell [...] le lira, y verra sans doute soi-même par transparence, mourra d'espoir aux premiers mots et recevra en dernières lignes une sorte d'adieu : "En quel futur plus sûr que ce mirage..." Très froid aujourd'hui, toujours. C'est le 4 février45. » Fin août 1929, Jean Paulhan propose d'envoyer à l'impression « Très haut amour... ». Sous l'aiguillon de cette lettre, Catherine Pozzi retravaille la mouture du poème envoyée en février et modifie notablement les deux dernières strophes. Début septembre, elle envoie à son interlocuteur la nouvelle version du poème, provisoirement nommé « Ave atque Vale ». Le dialogue avec Jean Paulhan, qui a précipité l'accouchement de la version définitive du texte, est également décisif pour le choix du titre. Dans une lettre du 12 octobre 1929, Catherine Pozzi renonce à « Ave atque Vale », suggère « Ave », se ravise et tranche en faveur de « Très haut Amour46 »... Un échange avec le rédacteur en chef de La NRF conduit

41. C. Pozzi, Cahier 1928-1929, fo $1 \mathrm{v}^{\circ}, \mathrm{BnF}$.

42. C. Pozzi, Journal 1913-1934, Paris, Ramsay, 1987, p. 476-477 (31 décembre 1928-1er janvier 1929).

43. Ibid., p. 485 (4 février 1929).

44. Voir fac-similé du poème envoyé dans Catherine Pozzi et Jean Paulhan, Correspondance 1926-1934, Paris, éditions Claire Paulhan, 1999, p. 190.

45. C. Pozzi, Journal 1913-1934, op. cit., p. 485 (4 février 1929).

46. Voir Catherine Pozzi et Jean Paulhan, Correspondance 1926-1934, op. cit., p. 91. 
finalement à adopter le titre d' « Ave ». L'ultime version de la dernière strophe du poème est plus hermétique que l'état rédactionnel de février 1929 :

Vous referez mon nom et mon image

De mille corps emportés par le jour,

Vive unité sans nom et sans visage,

Cour de l'esprit, ô centre du mirage

Très haut amour.

Les modifications successives du poème convergent vers une même fin : que s'efface la trace des pathétiques amours humaines et que seul demeure l'amour de l'Unique, vision d'une transcendance, « re-création mystérieuse au-delà du dogme catholique 47 ».

L'on observe que l'échange épistolaire donne à l'autre la possibilité d'intervenir au cours de la genèse - pensons seulement au choix du titre «Ave » pour le poème de Catherine Pozzi -, et dans certains cas de l'infléchir. Le journal, qui est d'abord un espace d'écriture solitaire, peut jouer dans la genèse d'une œuvre un rôle sensiblement différent de celui joué par la correspondance. D'une part, il peut tout accueillir au-delà ou en deçà d'une exigence de lisibilité, en dehors de toute perspective de communication : il est, plus que la lettre, l'espace propre aux balbutiements de la genèse ; d'autre part, plus que les lettres, disséminées au gré de leur envoi, le journal peut se prêter à la relecture et aux éventuels reprises et approfondissements, bref à l'exploitation des idées enregistrées et des formulations essayées.

Nous aurions souhaité démontrer que de bout en bout le journal tient sa place dans la genèse, jusque dans le cas où un journal personnel, maintenu dans son statut factuel, est transformé en livre. On peut considérer que le journal manuscrit ou tapuscrit est un élément constitutif de l'avant-texte du journal publié. Même si nous nous contentons de cas d'autoédition, autrement dit de cas où la leçon du journal publié a été déterminée par le diariste lui-même, l'on ne peut manquer de noter les transformations du journal (manuscrit ou tapuscrit) qui font de ce dernier, dans le cas d'une publication, une étape vers l'œuvre finale. La conversion à la publication exige maintes transformations, dictées à la fois par une nécessité de lisibilité, par un éventuel souci esthétique et par une considération d'impératifs moraux ou juridiques. Prenons pour seul exemple le journal de J. H. Lartigue.

En tête du manuscrit du journal de l'année 1924 s'inscrit un préambule, plein d'ombres :

1924

ici des pages. Écrites pour une seule raison : me guérir... ou tout at moins, me sottager. Ni un Journal, ni un livre ; elles ne sont qu'un MÉDICAMENT.

Ce dont je suis atteint étant une maladie sans nom : son remède n'a pas davantage à être baptisé. Supposition (invraisemblable autant que prétentieuse) : Si devenu « grand-peintre » un jour, plus tard, on essayait de les lire « pour voir un peu »... alors On aurait <alors> doublement tort.

$1^{\circ}$ Parce que ce que j'écris n'est pas une « chose à lire »
La phase

prééditoriale : place du journal dans la genèse de l'imprimé

47. P. Boutang, Karin Pozzi et la quête de l'immortalité, Paris, La Différence, 1991, p. 261. 
$2^{\circ}$ Parce que lorsque j'écris e'est que je suis malade, torturé ou gâteux et me précipite alors sur mon « remède ». Tandis que lorsque je peins ; c'est que j'ar qu'un trop plein de santé cherche à s'extérioriser.

Donc : au lieu de mieux faire comprendre, <loin d'expliquer> elles embrouilleraient tout. Un point - : c'est tout 48 .

L'écriture journalière répond à une nécessité thérapeutique, écriture de crise autodestinée. À l'attention d'un éventuel lecteur intrus (et c'est plus le lecteur posthume qu'anthume que semble viser Lartigue), le préambule apparaît comme une mise en garde qui invalide par avance la lecture du journal manuscrit. Les pages manuscrites journalières « embrouilleraient tout », brouilleraient l'image de l'homme heureux que Lartigue s'emploie à construire. Aussi, lorsque Lartigue a préparé, quelques décennies plus tard, l'édition d'une partie de son journal, il n'a pas retenu ce passage pour la version publiée, et il a soigneusement toiletté le journal dans la perspective de l'image qu'il souhaitait laisser de lui au lecteur, convertissant la friche journalière en un jardin extraordinaire, l'autoportrait, dont il a gommé les ombres, de l'homme heureux.

Le journal personnel, même s'il est un texte sans brouillon, même s'il subvertit la notion d'œuvre, le plus souvent associée à une visée téléologique, n'est nullement étranger à l'univers de la genèse. Il est susceptible d'intervenir à toutes les phases de la genèse, de la phase prérédactionnelle à la phase prééditoriale, possiblement associé selon les catégories de scripteurs à différentes étapes de la fabrication. Écriture en acte et en recherche, le journal est une merveilleuse pièce, multifonctionnelle, de l'outillage génétique.

48. Feuillet manuscrit au recto et au verso, non daté, qui figure en tête du journal de 1924. Le recto et le verso sont biffés. Code de transcription utilisé : <abc> : ajouté en interligne. Archives de l'Association des amis de J. H. Lartigue. Tous droits réservés. 
Françoise Simonet-Tenant, agrégée de Lettres modernes, est maître de conférences à l'université Paris XIII depuis 2000. Ses travaux portent sur le journal personnel et sur la correspondance (Catherine Pozzi et Jean Paulhan, Correspondance 1926-1934, Paris, éditions Claire Paulhan, 1999 ; Le Journal intime, Paris, Nathan, 2001 ; Journal personnel et correspondance (1785-1939) ou les affinités électives, Louvain-la-Neuve, Academia-Bruylant, 2009). Ses études de génétique ont porté en particulier sur Annie Ernaux, André Gide et Catherine Pozzi.

Françoise Simonet-Tenant, francoise.simonet-tenant6@orange.fr

\section{Le journal personnel comme pièce du dossier génétique}

Le journal personnel, même s'il subvertit la notion d'œuvre, le plus souvent associée à une visée téléologique, n'est nullement étranger à l'univers de la genèse. Loin d'être un simple discours d'escorte, il peut devenir un des supports où s'effectue la genèse d'une œuvre autre que lui-même. D'inscription de l'idée fulgurante à l'enregistrement d'images obsessionnelles et d'écritures provisionnelles, le journal est susceptible de jouer un rôle important dans la phase prérédactionnelle (cas d'HenriPierre Roché, de Jean Cocteau, de Michel Leiris). Les exemples d'André Gide et de Catherine Pozzi montrent que le journal a sa place également dans la phase rédactionnelle, constituant un véritable soubassement de la fiction romanesque ou un atelier d'écritures poétiques. De bout en bout, le journal est une pièce de l'outillage génétique, jusque dans le cas où un journal personnel, maintenu dans son statut factuel, est transformé en livre. On peut alors considérer que le journal manuscrit (ou tapuscrit) est constitutif de l'avant-texte du journal publié (cas de Jacques Henri Lartigue) et intervient à ce titre dans la phase prééditoriale.

Even if it subverts the "œuvre" concept, the private journal, usually combined with a teleological aim, has its place in the universe of genesis. Far from being a simple accompanying speech, it can become one of the bases for the genesis of a work other than itself. From the jotting down of a striking idea to the recording of obsessive images and provisional writing, the diary may well play an important role in the pre-editing phase (as for Henri-Pierre Roché, Jean Cocteau and Michel Leiris). André Gide and Catherine Pozzi are examples illustrating that a journal also plays a role in the editing phase, forming a true bedrock of novelistic fiction or poetic writing workshops. Through and through the diary is a genetics tool, even when the private journal, kept in its factual status, is transformed into a book. One can then consider that the manuscript (or typescript) diary is constituent of the published diary's "avant-texte" (such as in the case of Jacques Henri Lartigue), and in this respect is involved in the pre-publication phase.

Selbst wenn es den Werkbegriff unterläuft, der zumeist mit einer teleologischen Absicht in Verbindung gebracht wird, ist das Tagebuch keinesfalls ein Fremdkörper in der Welt der Textgenese. Weit davon entfernt, eine bloße diskursive Begleiterscheinung zu sein, kann es zu einem jener medialen Träger werden, auf denen sich die Genese eines Werkes, das mit ihm nicht identisch ist, vollzieht. Von der Inschrift der aufblitzenden Idee bis zur Aufzeichnung obsessiver Bilder und einstweiliger Schreibweisen kann das Tagebuch eine wichtige Rolle in der präredaktionellen Phase spielen (der Fall von Henri-Pierre Roché, von Jean Cocteau, von Michel Leiris). Die Beispiele von André Gide und Catherine Pozzi zeigen, dass das Tagebuch seinen Platz auch in der redaktionellen Phase hat, wo es einen wahrhaften Unterbau der Romanfiktion oder eine Werkstatt des poetischen Schreibens darstellt. Das Tagebuch ist integrativer Bestandteil des genetischen Instrumentariums, bis hin zu dem Fall, in dem das Tagebuch, seinen faktualen Status beibehaltend, in ein Buch verwandelt wird. Man wird also annehmen dürfen, dass das handschriftliche (oder typographische) Tagebuch für den avant-texte des publizierten Tagebuches konstitutiv ist (der Fall von Jacques Henri Lartigue) und in dieser Eigenschaft Eingang findet in die präeditoriale Phase.

El diario personal, aunque trastoca la noción de obra, asociada por lo común con un designio teleológico, no es ajeno en absoluto al universo de la génesis; lejos de constituir un simple discurso de escolta, puede transformase en uno de los soportes en el que se efectúa la génesis de otra obra distinta. De la inscripción de la idea fulgurante al registro de imágenes obsesivas y de escrituras provisorias, el diario puede jugar un papel importante en la fase prerredaccional (es el caso de Henri-Pierre Roché, Jean Cocteau o Michel Leiris). Los ejemplos de André Gide y Catherine Pozzi demuestran que el diario encuentra también su lugar en la fase redaccional, constituyendo un verdadero cimiento de la ficción novelesca o un taller de escrituras poéticas. De punta a punta, el diario forma parte de las herramientas genéticas, aun en el caso en que el diario personal, mantenido en su nivel factual, es transformado en libro. En ese caso, el diario manuscrito (o dactilografiado) puede ser considerado como un elemento constitutivo del pre-texto del diario publicado (como en el caso de Jacques Henri Lartigue) y, como tal, interviene en la fase pre-editorial.

O diário pessoal, mesmo quando subverte a noção de obra, associada geralmente a uma perspectiva teleológica, de modo algum é estranho ao mundo da génese. Longe de ser um simples discurso de acompanhamento, pode servir de suporte à génese de uma obra diversa dele mesmo. Desde a inscrição de uma ideia fulgurante até ao registo de imagens obsessivas e de escritas provisórias, o diário é susceptível de desempenhar um papel importante na fase pré-redaccional (casos de HenriPierre Roché, de Jean Cocteau, de Michel Leiris). Os exemplos de André Gide e de Catherine Pozzi mostram que o diário também tem um lugar na fase redaccional, em que tanto pode servir de fundamento à ficção romanesca como de oficina de escrita poética. O diário é, na totalidade, uma peça do instrumental genético, chegando-se ao ponto de um diário pessoal que conserva o seu estatuto factual se transformar em livro. Podemos assim considerar que o diário manuscrito (ou dactiloscrito) faz parte do antetexto do diário publicado (casos de Jacques Henri Lartigue) e intervem a esse títutlo na fase pré-editorial.

Il diario, anche se sovverte la nozione di opera, associata a un'intenzione e un fine, non è tuttavia estraneo all'universo della genesi. Lungi dall'essere un semplice discorso di scorta, può divenire uno dei supporti per la genesi di un'opera a se stante. Luogo dell'idea folgorante e della registrazione di immagini ossessive e di scritture provvisorie, il diario può avere un ruolo importante nella fase preredazionale (nei casi di HenriPierre Roché, Jean Cocteau, Michel Leiris). Gli esempi di André Gide e di Catherine Pozzi Mostrano che il diario è un oggetto dello strumentario genetico, fino al caso in cui un diario, mantenuto nel suo stato, è trasformato in un libro. Si può allora dire che il diario manoscritto, o dattiloscritto, è costitutivo dell'avantesto del diario pubblicato (come nel caso di Jacques Henri Lartigue) e interviene a questo titolo nella fase preeditoriale. 